\title{
Detecting Microsurgical Complications with ViOptix Tissue Oximetry in a Pediatric Myocutaneous Free Flap: Case Presentation and Literature Review
}

\author{
Steven D. Kozusko, MD ${ }^{1} \quad$ Uzoma B. Gbulie, MD, FACS ${ }^{1}$ \\ ${ }^{1}$ Department of Plastic Surgery, The University of Tennessee Health \\ Science Center, Memphis, Tennessee \\ J Reconstr Microsurg Open 2018;3:e8-e12. \\ Address for correspondence Uzoma B. Gbulie, MD, FACS, \\ Department of Plastic Surgery, The University of Tennessee Health \\ Science Center, 1068 Cresthaven Road, Suite 500, Memphis, TN \\ 38119 (e-mail: ugbulie@uthsc.edu).
}

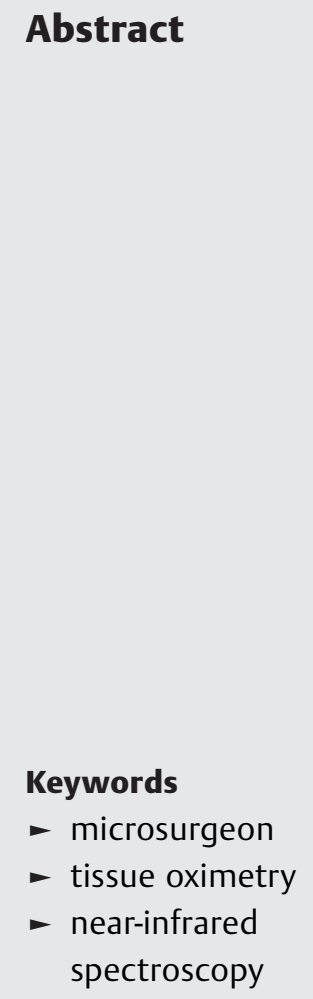

Background Microvascular compromise from arterial or venous occlusion is a common cause of free flap failure. The salvage rate following a microvascular compromise is dependent on detecting the problem early and intervening quickly.

Methods The ViOptix tissue oximeter measures tissue oxygen saturation using the nearinfrared spectroscopy technology. The ViOptix device has an alarm capability to warn of potential compromise to tissue perfusion. The tissue oximetry readings are visible on the bedside monitor and are relayed to a webpage link, which is accessible on a personal computer or mobile device, allowing real-time monitoring. This article presents a case where real-time monitoring allowed almost immediate detection of inadvertent pedicle compromise allowing flap salvage by repositioning without surgical intervention.

Results In the case presented, the patient's nurse inadvertently positioned a pillow under the location of the vascular pedicle likely causing microvascular compression. The ViOptix reading dropped and for this reason the nurse contacted the Plastic Surgery team. The drop was confirmed remotely and the flap was urgently evaluated in person. Once the pillow was removed, the ViOptix readings normalized and Doppler signals strengthened in the flap.

Discussion While tissue oximetry monitoring does not by itself ensure flap survival, it provides critical information than conventional flap monitoring would allow giving the microsurgeon the opportunity to make a quicker decision. ViOptix tissue oximeters are able to detect vascular compromise even before conventional clinical symptoms are present. Alas in several cases by the time clinical symptoms develop the flap may be beyond salvage.
Microsurgical techniques have allowed the performance of complex reconstructive endeavors with a good rate of success. Compromise of the arterial or venous anastomosis is commonly responsible for free flap failure. This most frequently occurs during the first 48 hours postoperatively. ${ }^{1}$ The early detection of microvascular complications improves flap salvage rate. ${ }^{2}$ Detecting the complication alone is insufficient if it is only detected after salvage is no longer feasible.
Therefore, ideal flap monitoring modalities should detect potentially catastrophic events early, allowing a microsurgeon an opportunity to salvage the flap.

Conventional free flap monitoring includes assessing the color, turgor, temperature, capillary refill, and arterial and venous Doppler signals with a handheld Doppler probe. The potential drawback with conventional monitoring techniques is that they may only detect the problem after it has progressed received

November 16, 2017

accepted

December 23, 2017
DOI https://doi.org/

10.1055/s-0038-1626728. ISSN 2377-0813.
Copyright $\odot 2018$ by Thieme Medical

Publishers, Inc., 333 Seventh Avenue, New York, NY 10001, USA. Tel: +1(212) 584-4662.
License terms

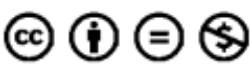


beyond the point of salvage. Qualities of ideal flap monitoring modalities include being noninvasive, quantitative, sensitive, generalizable, easy to interpret, providing real-time information, detecting early changes flap perfusion, and having minimal risks to the patient and flap. ${ }^{3,4}$ Near-infrared spectroscopy measures scattering and absorption of light at 700 to $100 \mathrm{~nm}$ wavelength. ${ }^{5}$ Tissue oximetry technology makes use of spectroscopy and the chromophores in human tissue to calculate tissue oxygen saturation as a measure of flap perfusion.

Herein, we present a unique case report of a pediatric patient who underwent a free vertical rectus abdominis myocutaneous (VRAM) flap for a right Gustilo-Anderson IIIb open tibial fracture sustained in an all-terrain vehicle (ATV) accident. The flap was monitored postoperatively with handheld Doppler as well as continuously with a ViOptix nearinfrared spectroscopy device (ViOptix T.Ox Tissue Oximeter, ViOptix, Freemont, CA). We highlight the utility in the ViOptix device detecting impending microvascular compromise of the flap from a positional change in the extremity, which resulted in external pedicle compression. The rapid detection of the problem prompted urgent flap evaluation and repositioning of the extremity by the plastic and reconstructive surgery resident, with subsequent recovery of tissue oximeter readings and avoidance of free flap compromise.

\section{Methods and Case Description}

Consent was obtained from the patient's father to document a description of the patient's reconstructive surgery course including the use of photography.

The patient is a 16-year-old boy who sustained a GustiloAnderson IIIb open tibial fracture to his right lower extremity from an ATV accident ( $\boldsymbol{- F i g . ~ 1 ) . ~ F r a c t u r e s ~ o f ~ h i s ~ t i b i a ~ a n d ~ f i b u l a ~}$ with periosteal stripping and extensive soft tissue loss resulted. He was initially treated with debridement and external fixator placement. The Plastic and Reconstructive Surgery Service was consulted for soft tissue coverage. The patient had anatomically small caliber anterior tibial vessels, which were occluded in the zone of injury. The vascular surgeon assisted with exposure of the posterior tibial vessels proximal to the zone of injury. The left VRAM free flap was selected on the basis of a preoperative CT angiogram and the perforators were marked with a handheld $(9 \mathrm{MHz})$ Doppler (-Fig. 2). The flap

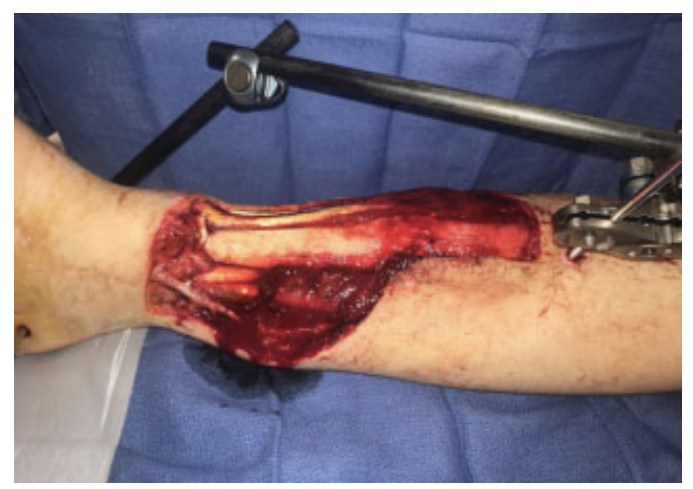

Fig. 1 Gustilo-Anderson IIIb injury with substantial soft tissue defect.

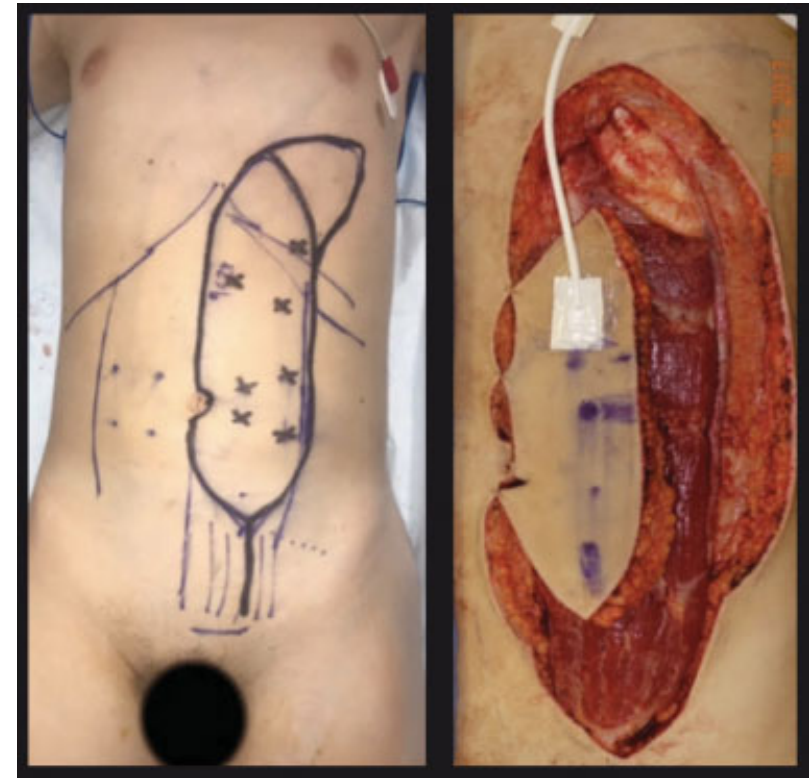

Fig. 2 Preoperative planning of the rectus abdominis myocutaneous free flap with the placement of the ViOptix probe prior to deep inferior epigastric vessel ligation.

was elevated in routine fashion preserving the dominant perforators. The ViOptix probe (ViOptix, Inc., Newark, CA) was placed on the flap skin paddle prior to ligation of the deep inferior epigastric vessels. The orthopedic surgery team performed the intramedullary nailing of the tibia while the flap perfused in situ with stable ViOptix readings.

Following this, the flap was harvested and an end-to-side arterial anastomosis was performed from the deep inferior epigastric artery to the posterior tibial artery. There was immediate brisk bleeding sustained from the larger one of the venae comitantes. This vessel was then anastomosed to the posterior tibial vein with a $3 \mathrm{~mm}$ coupler. With flow restored to the flap, arterial and venous signals were detected in multiple locations on the flap skin paddle as well as over the flap muscle. The flap was inset over the defect with exposed portions of the muscle covered with allograft. The ViOptix probe was placed on the distal aspect of the flap skin paddle (-Fig. 3 ) and had a

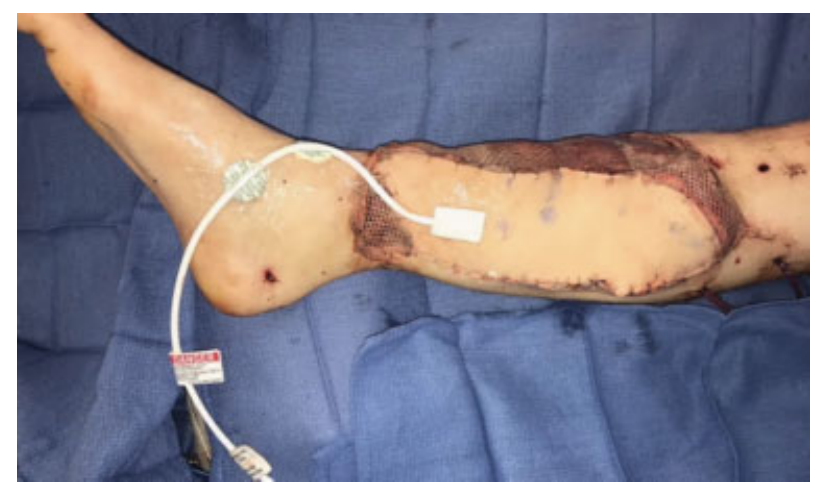

Fig. 3 Immediate postoperative placement of the ViOptix probe with the deep inferior epigastric vessel anastomosis at the proximal leg into the posterior tibial vessels. 
highest recorded value of $63 \%$ intraoperatively and maintained acceptable values immediately postoperatively, and the patient was transferred to the intensive care unit (ICU).

\section{Results}

The patient did well and was extubated immediately after surgery. He had no acute complications in the immediate postoperative period.

On postoperative day 1 , the resident evaluated the patient at $17: 02$ at which time the ViOptix read $71 \%$. Sometime thereafter, the ICU nurse performed a handheld Doppler check then repositioned the extremity to offset pressure on the patient's heel. In doing so, one of the pillows under the patient's distal extremity slid forward, compressing the pedicle. By 18:25 the reading dropped to $51 \%$ ( - Fig. 4), meeting the preset parameters on account of which a call was made to the Plastic Surgery team. The attending and resident assessed the ViOptix readings remotely on their cellular devices and called to mobilize the operating room for a possible flap exploration while heading into the hospital. The resident first assessed the patient and he had hardly discernible arterial and venous Doppler signals. He noted the new position of the pillow and repositioned the extremity with rapid improvement in the ViOptix reading as well as in the quality and volume of the flap Doppler signals. By 18:50 the flap's ViOptix was improving after repositioning, and by 19:00 the perfusion had returned close to the baseline (-Figs. 5 and $\mathbf{6}$ ).

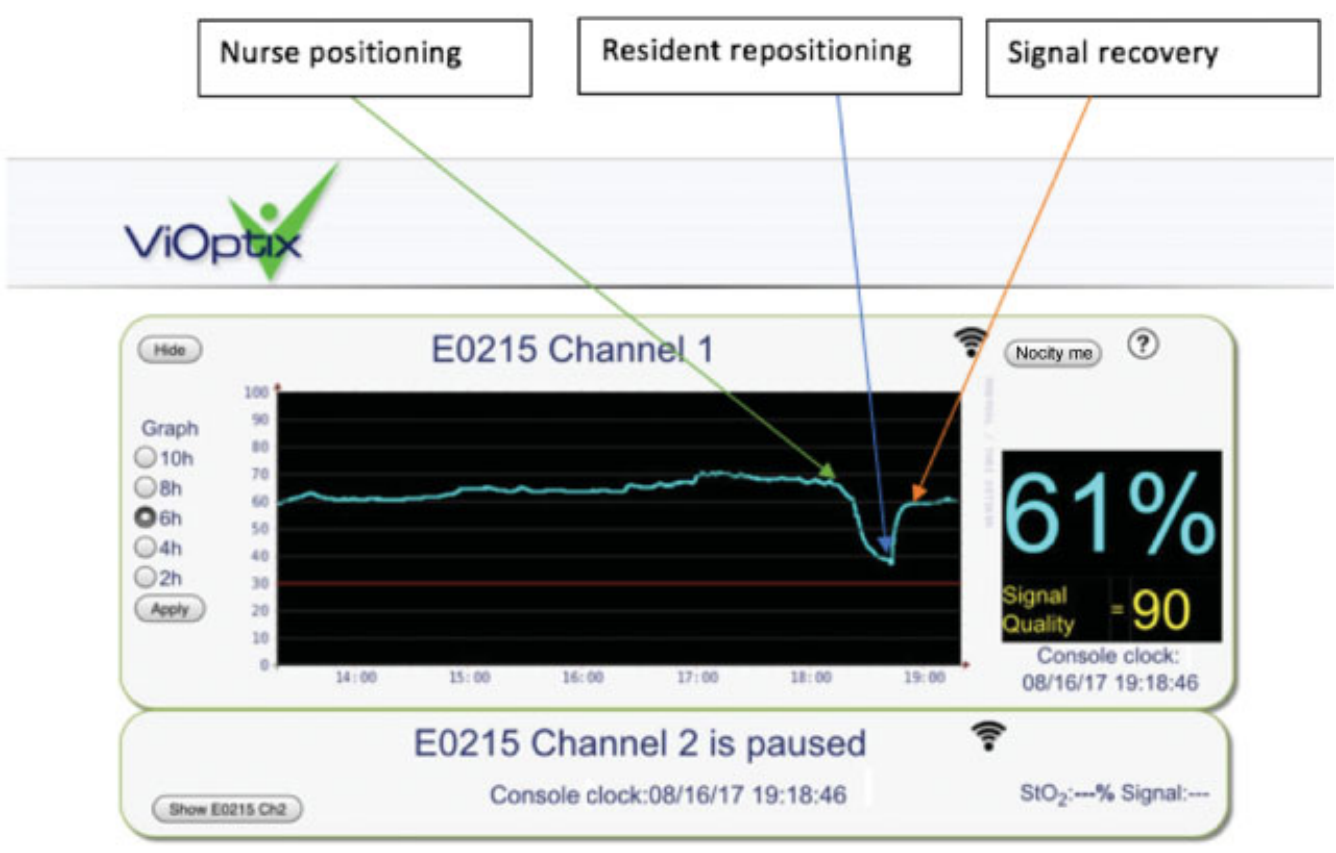

Fig. 4 Acute drop in ViOptix more than 20\% in less than 1 hour depicting time of nurse repositioning, time of resident repositioning, and time of recovery with corresponding ViOptix readings.

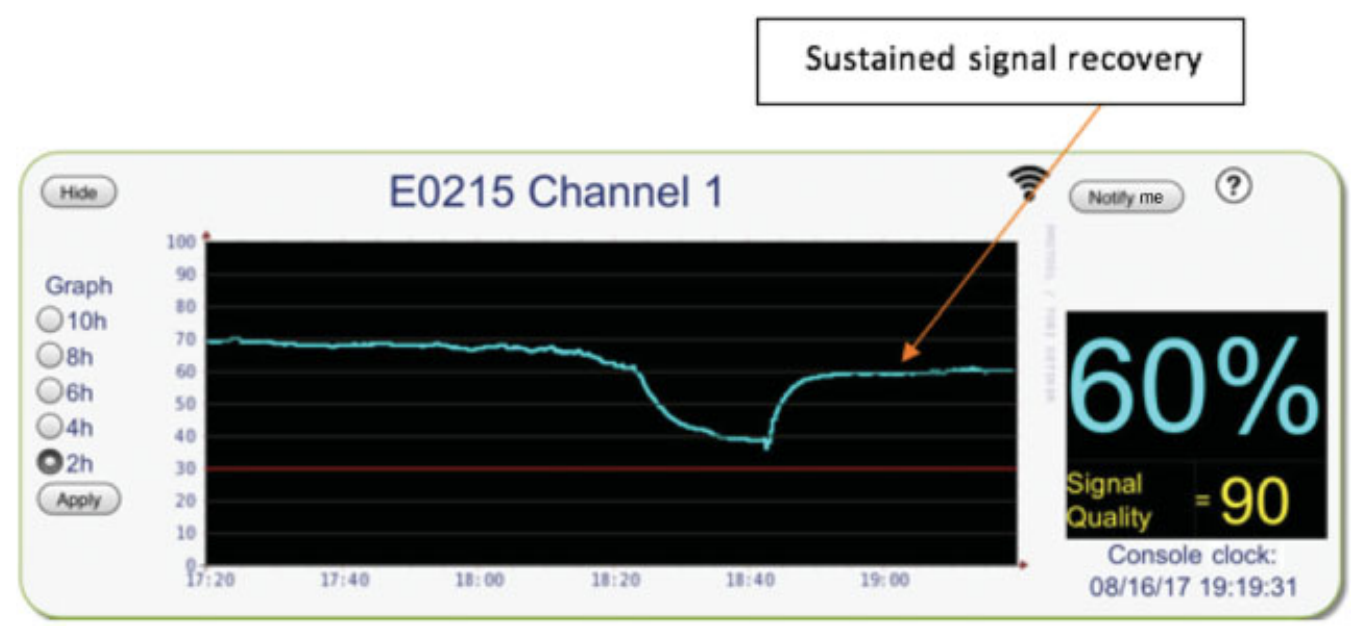

Fig. 5 Sustained recovery as seen on ViOptix reading after repositioning the leg to relieve pedicle compression. 


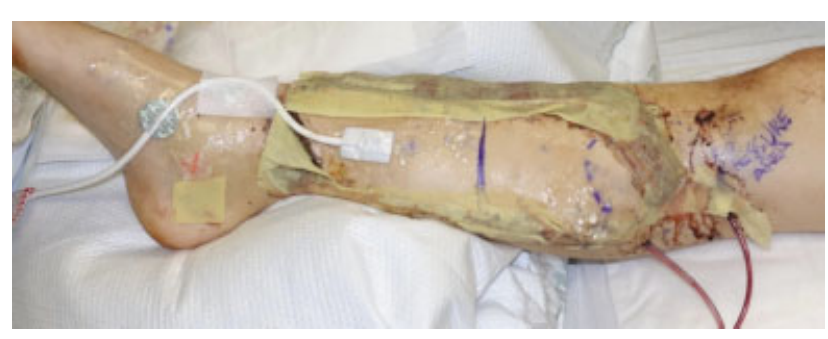

Fig. 6 Position of leg without compression to the pedicle.

The patient had normal ViOptix readings the remainder of postoperative day 1 with normal color, turgor, capillary refill, and Doppler signals. He went on to have an uneventful subsequent postoperative course. On postoperative day 7 , the ViOptix probe was removed and extremity dangling commenced on postoperative day 9 . He was discharged on postoperative day 14 . His extremity retained full sensation, plantar flexion, and dorsiflexion. His only functional limitation was absence of extensor hallucis longus function sustained during the initial injury. At follow-up, he continues to do well and is working with physical therapy on active range of motion.

\section{Discussion}

Microvascular compromise most commonly results from kinking, twisting, compression, or thrombosis of the pedicle. Other potential causes include a tight closure, positional changes, prolonged vasospasm, and hematoma. ${ }^{6}$ Anastomotic thrombosis is a devastating and at times unavoidable potential complication of free flap microvascular surgery. ${ }^{7}$ Earlier detection of problems with more timely intervention prevents the progression to microvascular thrombosis. ${ }^{8}$ The ViOptix tissue oximeter measures tissue oxygen saturation using the near-infrared spectroscopy technology. The device measures the scatter and absorption of near-infrared light by the principle chromophore in the skin, which is hemoglobin, with minor contributions from cytochrome c oxidase. ${ }^{5}$ The wavelength for near-infrared laser light is 700 to $100 \mathrm{~nm} .^{7}$ Near-infrared light is scattered due to the heterogeneous nature of human tissue. ${ }^{3}$ As hemoglobin selectively absorbs the near-infrared light, there is variation in the reflected scatter intensity and this is detected by the device. These data are a measure of the tissue oxygen saturation, which is an indirect indication of flap perfusion and congestion. The device itself has a sensor connected to a console by a fiberoptic cable. The external sensor is part of a $25 \mathrm{~cm}^{2}$ adhesive pad, while there is an intraoral sensor housed in a silicon block. The laser light sources and photodetectors measure up to a depth of $10 \mathrm{~mm} .{ }^{9}$ The ViOptix device has a built-in alarm that is triggered by a drop in $20 \%$ points within an hour or below an absolute value of $30 \%$ tissue oxygen saturation. ${ }^{9}$ The tissue oximeter can be monitored remotely in real time via a webpage link accessible on a personal computer or a mobile device.

Tissue oximetry provides critical information and warnings to a discerning microsurgeon who can then make a quicker decision than conventional flap monitoring would allow.
Conventional flap monitoring, the current gold standard, is not without its own challenges. Monitoring the flap's color, capillary refill, turgor, and Doppler signals is subjective, relying on the individual's experience with flap monitoring. To make a clinical determination of a flap's perfusion status, this person must be able to differentiate normal from pathologic. Typical postoperative protocols involve flap monitoring every $30 \mathrm{~min}$ utes or 1 hour during the first 24 to 48 hours followed by every 2 hours for the subsequent 24 hours, and so on. Flap salvage rates are directly related to the time that elapses from complication to detection. ${ }^{9}$ ViOptix tissue oximeters are able to detect vascular compromise before conventional clinical symptoms are present, which may coincide with irreversible compromise of the flap. Flap perfusion, and hence compromise, is detectable by noninvasive near-infrared spectroscopy in real time. Even though a flap may be evaluated frequently by the nursing staff using conventional means, the practical benefit of this technology goes further by allowing real-time monitoring by all team members including the nurse at bedside as well as remotely by the microsurgeon, fellow, resident, and other team members.

Other advancements in free flap monitoring include color duplex sonography, microdialysis, and laser Doppler flowmetry. ${ }^{10}$ The implantable Doppler is invasive, color duplex sonography requires a radiologist along with a microsurgeon for interpretation, microdialysis requires time and laboratory analysis, and laser Doppler flowmetry is operator dependent.

In the case presented, a pillow was inadvertently moved causing compression of the vascular pedicle (most likely the venous limb). The ViOptix monitor alerted the nurse to the change prior to clinical manifestations of flap compromise. This prompt identification of a problem led to evaluation of the flap within minutes of onset resulting in the urgent nonsurgical intervention of repositioning to relieve the pedicle compression. This saved the flap without the morbidity of operative exploration, thus preventing a potentially catastrophic complication. This is a unique instance of positional compromise in a pediatric patient monitored in real time using the ViOptix technology.

Many studies have evaluated the efficacy of tissue oximetry in microsurgical procedure. One case study describes using a saphenous vein stoma for mechanical leeching with monitoring of congestion using the ViOptix device. ${ }^{10}$ In a case series by Steele of 208 flaps in 145 patients undergoing autologous tissue perforator free flap breast reconstruction, five patients exhibited complications that were predicted using this device and no flap loss occurred. ${ }^{9}$ Keller evaluated 614 consecutive microsurgical flaps, using conventional methods to monitor the first 380 flaps and then included tissue oximetry for the subsequent 234 patients. ${ }^{8}$ They had no statistically significant difference in rates of flap reexploration, but the flap salvage rate increased from $57.7 \%$ without tissue oximetry to $93.75 \%$ with the technology. Steele evaluated 128 free flaps in a 3-year period with a statistical difference in the length of stay and flap survival in the tissue oximeter cohort. Scheufler et al used tissue oximetry to detect changes in tissue hemoglobin content and oxygenation in pedicled transverse rectus abdominis flaps. ${ }^{11}$ In a second series, these authors evaluated continuous 
tissue oxygen tension with near-infrared spectroscopy intraoperatively and postoperatively, noting that the measurements correlated with iatrogenic surgical discontinuation of vascular inflow. ${ }^{12}$ Repez et al found that near-infrared spectroscopy detected an abrupt decrease in tissue oxygen saturation due to arterial thrombosis before clinical signs were evident in 50 free flaps for autologous breast reconstruction. ${ }^{13}$

The learning curve for ViOptix tissue oximetry is not steep. Koolen et al studied the learning curve of using this technology in flap monitoring and found that as the curve improved, the rate of early detection increased and more flaps were salvaged. ${ }^{3}$ In their study, they also reported common pitfalls for the novice user. Striae on the skin are prone to insufficient signal strength, intense operating room lighting may interfere with analysis, and placing the probe directly over a vessel does not appropriately monitor perfusion of the flap tissue. This technology has been shown to influence clinical decision making. Bellamy et al showed that surgeons were significantly more likely to return to the operating room when tissue oximetry data were concerning, more so than based on the standard $8 \mathrm{MHz}$ Doppler. ${ }^{14}$

The biggest limitation of using tissue oximetry is the cost related to fiberoptic probes and monitor. ${ }^{8}$ The estimated cost of a tissue oximetry probe is $\$ 700$ to $\$ 1200$ with consoles costing about $\$ 30,000 .^{15,16}$ Another limitation is the depth of penetration and need for placement of the adhesive pad on the flap. The depth of penetration for the ViOptix tissue oximeter is $10 \mathrm{~mm}$. There are cases where the flap is buried and there is no location to place the pad. Furthermore, experience is needed to learn the optimal placement of the sensor with different flaps. These limitations are acceptable compared with those involving the other technologies available including the invasive nature of implantable Doppler systems and the reliance on expertise staff with color duplex sonography.

\section{Conclusion}

Tissue oximetry is a noninvasive yet sensitive, reliable, and easy to implement flap monitoring modality. ${ }^{7}$ Increased salvage rate of flaps monitored with tissue oximetry has been demonstrated in the literature. We present a unique case of a pediatric trauma patient where real-time remote monitoring and ViOptix alarming alerting the microsurgical team to the early development of a potentially catastrophic complication. This technology is clearly an essential tool in the armamentarium of the microsurgeon with practical benefits to flap monitoring and indeed overall patient care.
Conflict of Interest

None.

\section{References}

1 Wei FC, Mardini S. Flaps and Reconstructive Surgery. 2nd ed. Chinca: Elsevier Health Sciences; 2016

2 Khouri RK, Cooley BC, Kunselman AR, et al. A prospective study of microvascular free-flap surgery and outcome. Plast Reconstr Surg 1998;102(03):711-721

3 Koolen PG, Vargas CR, Ho OA, et al. Does increased experience with tissue oximetry monitoring in microsurgical breast reconstruction lead to decreased flap loss? The learning effect. Plast Reconstr Surg 2016;137(04):1093-1101

4 Keller A. Noninvasive tissue oximetry for flap monitoring: an initial study. J Reconstr Microsurg 2007;23(04):189-197

5 Colwell AS, Craft RO. Near-infrared spectroscopy in autologous breast reconstruction. Clin Plast Surg 2011;38(02):301-307

6 Lin SJ, Nguyen MD, Chen C, et al. Tissue oximetry monitoring in microsurgical breast reconstruction decreases flap loss and improves rate of flap salvage. Plast Reconstr Surg 2011;127 (03):1080-1085

7 Bank J, Beederman M, Shore AM, Song DH. Mechanical leeching with venocutaneous fistula and monitoring with near-infrared spectroscopy. Plast Reconstr Surg Glob Open 2013;1(07):e56

8 Keller A. A new diagnostic algorithm for early prediction of vascular compromise in 208 microsurgical flaps using tissue oxygen saturation measurements. Ann Plast Surg 2009;62(05):538-543

9 Steele MH. Three-year experience using near infrared spectroscopy tissue oximetry monitoring of free tissue transfers. Ann Plast Surg 2011;66(05):540-545

10 Lin CM, Huang CC, Hsu H, Chiu CH, Chien SH. Advancements in free flap monitoring in the last decade: a critical review. Plast Reconstr Surg 2010;126(02):679, author reply 679-680

11 Scheufler O, Exner K, Andresen R. Investigation of TRAM flap oxygenation and perfusion by near-infrared reflection spectroscopy and color-coded duplex sonography. Plast Reconstr Surg 2004;113(01):141-152, discussion 153-155

12 Colwell AS, Wright L, Karanas Y. Near-infrared spectroscopy measures tissue oxygenation in free flaps for breast reconstruction. Plast Reconstr Surg 2008;121(05):344e-345e

13 Repez A, Oroszy D, Arnez ZM. Continuous postoperative monitoring of cutaneous free flaps using near infrared spectroscopy. J Plast Reconstr Aesthet Surg 2008;61(01):71-77

14 Bellamy JL, Mundinger GS, Flores JM, et al. Do adjunctive flapmonitoring technologies impact clinical decision making? An analysis of microsurgeon preferences and behavior by body region. Plast Reconstr Surg 2015;135(03):883-892

15 Smit JM, Zeebregts CJ, Acosta R, Werker PM. Advancements in free flap monitoring in the last decade: a critical review. Plast Reconstr Surg 2010;125(01):177-185

16 Pelletier A, Tseng C, Agarwal S, Park J, Song D. Cost analysis of near-infrared spectroscopy tissue oximetry for monitoring autologous free tissue breast reconstruction. J Reconstr Microsurg 2011;27(08):487-494 